\title{
Research on the Fusion of Power Grid Data and Urban Facilities Data
}

\author{
Jing Tian ${ }^{1, *}$, Guan $\operatorname{Lan}^{1}$, Li Jin ${ }^{1}$, Guo Yanxia ${ }^{2}$, Li Ao-sen ${ }^{2}$, Zhang Youfu ${ }^{2}$, and Yu Zhuangzhuang ${ }^{2}$ \\ ${ }^{1}$ State Grid Economic and Technological Research Institute CO.LTD.Beijing, China \\ ${ }^{2}$ State Grid Corporation of China. Beijing, China
}

\begin{abstract}
The three-dimensional design of power grid infrastructures needs to consider their adjacent urban facilities, including industrial and civil buildings and tunnels, roads, pipelines, and etc. Therefore, in order to realize the integration of the three-dimensional design model of the power grid and the BIM model of those urban infrastructures, this paper attempts to establish the information model classification and coding method to unify the representation methodology of concepts and technologies of multi-industry infrastructures. The developed data format conversion method and data lightweighting method can be utilized to better integrate the three-dimensional design results of the power grid project with the BIM data of the urban infrastructures, and then these digitalized results of the multi-industry facilities can be used to improve the life-cycle management of the power grid engineering projects.
\end{abstract}

\section{Introduction}

The management and application of three-dimensional design on transmission and transformation project is the key link to supporting the construction of the new power system strategies. Since 2017, China has started the first batch of projects with three-dimensional design, including $3 \mathrm{UHV}$ AC projects and $1 \mathrm{UHV}$ DC project [1].The experimental construction has basically realized the depth of construction drawing design in transmission and transformation, by using three-dimensional design. With the deepening of the research, under the background of constructing new power system with new energy, the access of massive data and the change of traditional design have brought brand-new problems to be solved [2].

In actual projects, it may introduce potential errors when team members with different modeling tools are trying to move models from one platform to another, or merge multiple models. An effective way to reduce errors is to introduce a unified standard in model-data exchange. Based on the review of the current classification and coding standards in BIM models, this paper sort and summarize a series of standards, including BS ISO 12006-22015, GB/T 51269-2017, and Q/GDW 11809-2018. Especially, this paper comes up with a method of connecting the information model code in the substation construction project with international standards and national standards [3].

\section{Related research at home and abroad}

The three-dimensional design is developed from the United States and gradually extended to developed countries such as Europe, Japan and South Korea. At present, the development trend and application level of three-dimensional design in these countries have reached a certain extent. The United States is the most widely used and in-depth. In the United States, the application of BIM has begun to take shape. Major design firms, construction companies and owners have taken the initiative to apply BIM in their projects. Government and industry associations have also introduced various BIM standards [4].

In Japan and South Korea, BIM applications have expanded nationwide and have risen to the level of government advancement. In March 2010, Official Office Repair Departments of the Ministry of Land, Infrastructure, Transport and Tourism announced that it would promote BIM technology in its jurisdictional construction projects and implement BIM applications based on design operations [5-6].

In recent years, domestic design units have carried out explorations on the digital design of substation, coming up with the standard of Grid Information Model(GIM). The State Grid has compiled a data format standard for interaction of GIM format, however, in the context of digital power grids and smart city, how to combine the power grid models and urban facilities models, still remains to be explored.

\section{The classification method of urban facility model and power grid model}

\subsection{A.Urban Facility Model}


Urban facilities is modeled with BIM standards, which follow the IFC standard system. The information classification and coding system related to the construction industry in China, mainly includes the product information system and the constructiondesigning information. These classification systems are divided according to the principle of engineering projects. They are compiled differently, and the application scopes are also different. Information classification methods are mainly divided into three types: line classification, area classification and hybrid classification.

The line classification divides the objects into several levels according to selected attributes (or characteristics), and each level is divided into several categories. Categories at the same level in the same branch constitute a parallel relationship, and categories at the different levels constitute a subordination relationship. Categories at the same level are deplicated each other. By using line classification, it's easier to read, understand and deliver the information. At the same time, the disadvantage is that the classification structure is difficult to change. The coding length will be longer with more classification levels, which means data processing will be more difficult.

The area classification method treats several attributes or characteristics of the selected classification object as several area, each of which can be divided into independent parts. Then the area is arranged in parallel with order. Category in area can be combined with a category in another area. The advantage is that the area are independent. Any changes in one area will not affect other area, and it has better flexibility and adaptability. The disadvantage is that it wastes the capacity of the code, and the category combination is complicated. It is difficult to manually process information.

Hybrid classification is the comprehensive use of area classification and line classification. Its advantages are that the classification structure is flexible and efficient. It has better hierarchical relationships and clear logical relationships. The disadvantage is that the grouping structure of the category is more complicated, and it is difficult to manually process information.

According to the "Building Information Model Classification and Coding Standards", while referring to the OmniClass classification method, the substation adopts a combination of surface classification and line classification. "Component" and "attribute" are the relationship of the surface, in each sub-table, Using the line classification method to divide the classification objects into four levels: large, medium, small and fine. The coding structure should include table code, major category code, medium category code, small category code and detailed category code. All levels of codes should be represented by 2 Arabic numerals.

\subsection{B.Power Grid Model}

The substation contains a variety of construction facilities such as fences, lawns, roads, main control rooms, power distribution rooms, etc.; in addition, there are various supporting auxiliary facilities such as steel frames, outgoing poles and towers. The main electrical equipment in the substation includes electrical equipment such as busbars, transformers, breakers, switches, current transformers, post insulators, voltage transformers, reactors and capacitors [7].

In order to meet the needs of the three-dimensional design of power transmission and transformation projects, the State Grid issued "Q/GDW11809-2018: Transmission and Transformation Engineering Threedimensional Design Model Interaction Specification" and proposed the engineering model level in the specification. Different from "GB/T 512692017:Building Information Model Classification and Coding Standard" and "BS ISO 12006-22015", the information classification structure is based on the definition and attribute level as the classification principle. The engineering model level is based on the whole station level, unit level, system level, At the equipment level and component level, a single building is dismantled, and the substation is dismantled from the substation level of each component space.

\section{Transformation between IFC model for urban facilities and GIM model for power grid projects.}

Referencing related research results of automatic conversion between different programming languages in software engineering, a specific conversion method between IFC and GIM can be proposed. After the BIM model of urban buildings and facilities is streamlined and certain information conversion (including the conversion of coordinate system, graphic information and non-graphic information), it is imported into the three-dimensional design of the power grid project as the environmental elements of the planned power grid project; the three-dimensional design process data of the power grid project Lightweight, exported in IFC file format and integrated into the BIM model of urban buildings and facilities, as an important part of the city's three-dimensional model.

In the specific work of classifying power grid engineering information models, the relationship between the systematicness and practicality of classification should be handled flexibly. The classification and coding standards for power grid projects are mainly to handle such relationships. For example, for a classification system, the lower-level category and the upper-level category should be the same type of object, and the lower-level category and the upper-level category should be a subdivision relationship on the object category. This is the compilation of the general classification system. Principle, this is related to the systematic nature of classification; on the other hand, engineers and technicians are more accustomed to decomposing the work content into smaller work content, so that a decomposition relationship is formed between the lower-level categories and the upper-level categories. Rather than category breakdown relationships. Therefore, when classifying power grid 
engineering information models, it is necessary to reasonably balance the systematicness of classification and the practicality of decomposition according to factors such as the frequency of use of classification and coding in typical projects, and put forward the classification and coding standards of power grid engineering information models pragmatically .

Therefore, according to the classification and description of "GBT 51269-2017 Building Information Model Classification and Coding Standard" and "BS ISO 12006-22015", the information model coding of the substation should start with the engineering level model, and according to the engineering level model, the whole station level and unit level, System level, equipment level (including equipment components), first check whether there is a corresponding classification in the "GBT 51269-2017 Building Information Model Classification and Coding Standard" promulgated by the Ministry of Housing and Urban-Rural Development. If there is no such classification, you need to follow The hybrid classification method supplements the corresponding classification and further encodes the supplementary classification.

In summary, taking the surface classification method as an example, the transformation of urban facilities to the power grid information model can be completed according to the following steps:

(a) Sort out the design and construction specifications of power grid projects: collect the commonly used design and construction of power grid projects, and sort out the related and restrictive provisions between the power grid project and the surrounding urban environment and natural environment;

(b) Express the relationship between power grid engineering and urban built environment: further standardize the relationship and constraint relationship between power grid engineering and urban built environment;

(c) Model content extraction (simplification): In order to protect the intellectual property rights and commercial secrets of the three-dimensional design of the power grid project, at the same time, simplify the BIM model of the city's buildings and facilities to realize the lightweight of model data. Therefore, it is necessary to study the method of model simplification (lightweight) export;

(d) Sort out the IFC standard and GIM standard: sort out the language elements and grammar in the IFC standard, as well as the semantic understanding in the BIM context, and cooperate with the previous research on the GIM standard to prepare for the later establishment of the mapping between GIM and IFC;

(e) Study the mapping relationship between IFC and GIM representation schemes: From the perspective of domain-specific languages, analyze the mapping relationship between the description elements of the IFC format and the GIM format, and then study the IFC data format and GIM data format of the BIM model on this basis Compatible method;

(f) Propose a compatible representation method between IFC model and GIM model: Based on the power grid engineering domain ontology, propose a general description framework that can describe two different representation schemes of IFC and GIM;

(g) Format conversion of IFC and GIM files: Refer to the related research results of automatic conversion between different programming languages in software engineering, and propose a specific conversion method between IFC and GIM. After the BIM model of urban buildings and facilities is streamlined and certain information conversion (including the conversion of coordinate system, graphic information and non-graphic information), it is imported into the three-dimensional design of the power grid project as the environmental elements of the planned power grid project; the threedimensional design process data of the power grid project Lightweight, exported in IFC file format and integrated into the BIM model of urban buildings and facilities, as an important part of the city's threedimensional model.

\section{Conclusion}

There are two common grounds between the power grid projects and urban facilities: both of them have largescale and complex systems, and the construction and service cycle is long. Multiple organizations need to adopt a variety of different professional software for information transmission and sharing. It is necessary to establish a series of industry-recognized standards, in order to achieve the interoperability of digital results in the project life cycle management. There are three important standards proposed by Building Smart:IFC,IFD and IDM. These standards later become the base of BIM standard system. At the same time, The State Grid Corporation of China developed the GIM format as a standard for the interaction of threedimensional design models. However, the standards of BIM and GIM are not completely unified, and there are problems in mutual conversion, which affects the establishment of a unified urban planning or a geographic information system for a power grid model. This paper studies the classification and modeling methods of urban facilities and power grid projects models, and establishes the internal mapping relationship of the model. At the same time, transformation method between urban facilities and power grid projects is expounded. For urban facilities, the mapping relationship of the area classification method can be used to convert the corresponding BIM standard model components into model components that conform to the GIM standard, in order to achieve the purpose of model conversion.

This work was supported by the State Grid Corporation's S\&T project ' Research on Key Technologies of Three-Dimensional Design and Engineering Data Support Based on New Generation Power System' (No.SGZJJY00SJJS1900119), China. 


\section{References}

1. Zhang H F Cheng X J and Shi Y T 2011 Study on 3D Modeling for History Building and Precision Analyzing pp 471-476.

2. Yang G W, Xu J, Sun K 2014 Discussion the Strategy of Indoor 3D Fine Modeling Applied Mechanics \& Materials vol 536-537:1598-1602.

3. Das M 2015 Social bimcloud: a distributed cloudbased bim framework for object-based lifecycle information exchange and supply chain integration Visualization in Engineering vol 3(1) pp 1-20.

4. Faraj I, Alshawi M and Aouad G 2000 Industry foundation classes Web-based collaborative construction computer environment: WISPER Automation in Construction vol 10(1) pp 79-99.
5. Pazlar T and Turk Z 2008 Interoperability in practice: Geometric data exchange using the IFC standard Electronic Journal of Information Technology in Construction vol 13 pp 362-380.

6. Dakai S, Xin Q, Junhui H, Zhili W and Lizhong Q 2013 Conducting Research and Development of Grid Information Model (GIM) Technology and Constructing the Sharing Platform of Smart Grid Technology Information Electric Power Construction vol 34(8).

7. Jiacheng, H. Qian, A. I. Xueyuan, D. Binai, L. I. \& Zhengyu, L. U. "Feasibility Study of Substation Digital Handover Based on IFC4 Data Standard," Electrical \& Energy Management Technology, 2014. 